\title{
Iris based Human Identific ation using Median and Gaussian Filter
}

\author{
Geetanjali Sharma ${ }^{1}$ and Neerav Mehan ${ }^{2}$
}

\begin{abstract}
Biometric traits based system gives an automated recognition for a person based on unique features of an individual. Iris recognition is regarded as the most reliable and accurate automated recognition system as it is a safe body part and does not change with time. This paper presents a novel approach for iris based recognition system based on median filter and compares it with another existing approach based on Gaussian filters. The results show that the proposed method is better than the previous ones. Accuracy of the proposed system is $99.07 \%$.
\end{abstract}

Keywords: iris recognition, biometric identification, pattern recognition

\section{Introduction:}

Biometric system has been used widely by both the government and private entities in for effective security and authentication systems. Many biometric traits are used for to accomplish this, such as finger print, face, ears, iris, and palm print. Among them, iris based recognition is commonly recognized as one of the most reliable biometric measures [1]. The reason to this is that, iris has a random morphogenesis and it has no genetic penetrance. Moreover, iris morphology remains stable through all human life, also the probability for two similar irises on distinct persons at $1 / 10^{72}$ [1].

Iris is composed of elastic connective tissues such as trabecular meshwork. The agglomeration of pigment is formed during the first year of life, and pigmentation of the stroma occurs in the first few years [4][5]. The highly randomized appearance of the iris makes its use as a biometric well recognized.

The iris based human recognition is a relatively young approach. The first iris based recognition system came into existence in 1994. Iris color is often found by the density of melanin pigment in its anterior layer and stroma. For instance if there is a blue irises which results from absence of pigment in it, long wavelength light penetrates through it which is then absorbed by the pigment epithelium, on the other hand, short wavelength rays are reflected back and scattered by the stroma [2].

Median Filter: In median filtering, the neighboring pixels are ranked according to their intensity, and the median value is calculated, which becomes the new value for the central pixel.

\footnotetext{
${ }^{1}$ BUEST

${ }^{2}$ BUEST
} 
Median filters can perform very well in rejecting certain types of noise, in which some individual pixels have extreme values. In the filtering operation, the pixel values in the neighborhood window are ranked according to intensity, and the middle value (the median) becomes the output value for the pixel under evaluation [9].

\section{Related Work:}

Vanaja Roselin.E.Chirchi [3] gives a paper in which she has focused on an efficient methodology for recognition and verification for iris detection, even when the images have some obstructions, visual noise and different levels of illumination. She has used the CASIA iris database to accomplish her work, it will also work for UBIRIS Iris database which has images captured from distance while a person is not still. Efficiency is acquired from iris detection and recognition when its performance evaluation is accurate.

Yngzi Du and others [6] have proposed an iris based human detection system using priori pupil identification. The captured iris image is then transformed into polar coordinates and the outer boundary of the iris is identified as the largest horizontal edge resultant by using Sobel filtering. Although the given approach has some demerits as it may fail in case of non-concentric iris and pupil, as well as for very dark iris textures.

J. Mira, and J. Mayer [7] on the other hand have applied the morphologic operators to obtain iris borders. They have detected the inner border if iris by applying threshold, opening and image closing and the outer border with threshold, closing and opening sequence.

Jaemin Kim, Seongwon Cho, and Jinsu Choi [8] have considered the assumption that the image captured intensity values can be well represented by a mixture of three Gaussian distribution components. Based on this assumption they have proposed a system which uses the Expectation Maximization algorithm to measure the respective distributions parameters. They have expected that 'Dark', 'Intermediate' and 'Bright' distributions consist the pixels corresponding to the pupil, iris and reflections areas respectively.

Previous Work: The figure below shows the flow chart for the work performed in the given paper.

Input Image: this is the test image which is used as an input image in the system. This image is to compared with the dataset present in the system. So, to compare it with dataset some changes has to made like normalization, RGB to Gray, histogram equalization. If the image is already present in Grayscale format then no need to convert it but if it is in RGB format then it should be converted to Grayscale format.

Crop image: Input image is cropped to get the iris for recognition. First of all the image is cropped and after this the filters are applied for noise removal and image enhancement for better feature extraction. 


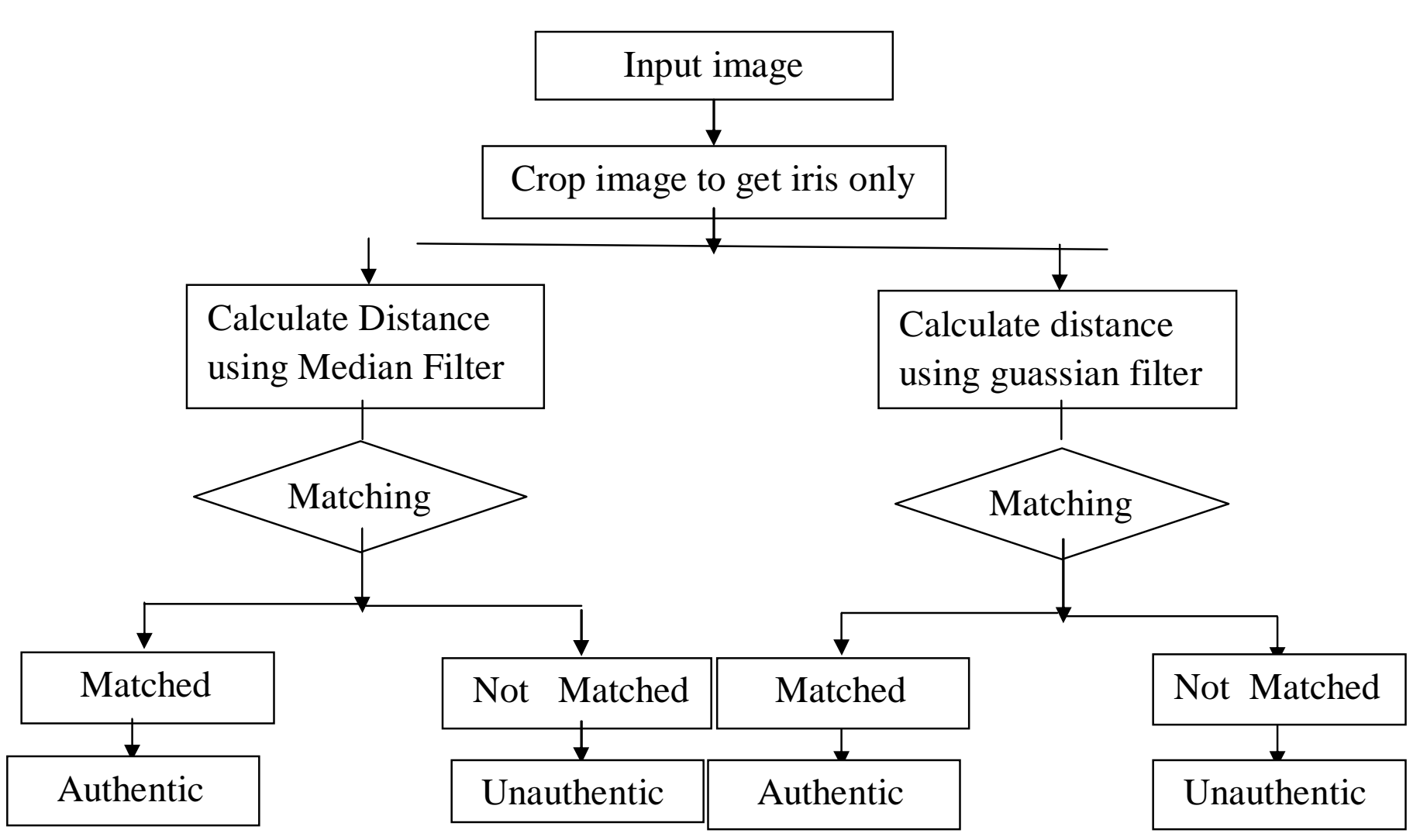

Fig. 1. Work flow diagram

Matching: Matching is done after feature extraction of image using SURF feature extractor and Euclidean distance is calculated. On behalf of which decision is taken whether image is matched or not. This helps us in finding the authentic person. So, to check authenticity we have used FRR curve against threshold value.

Results: The figures 2, 3, 4 and 5 show the results of various compared metrics between the previous approach and the proposed system.

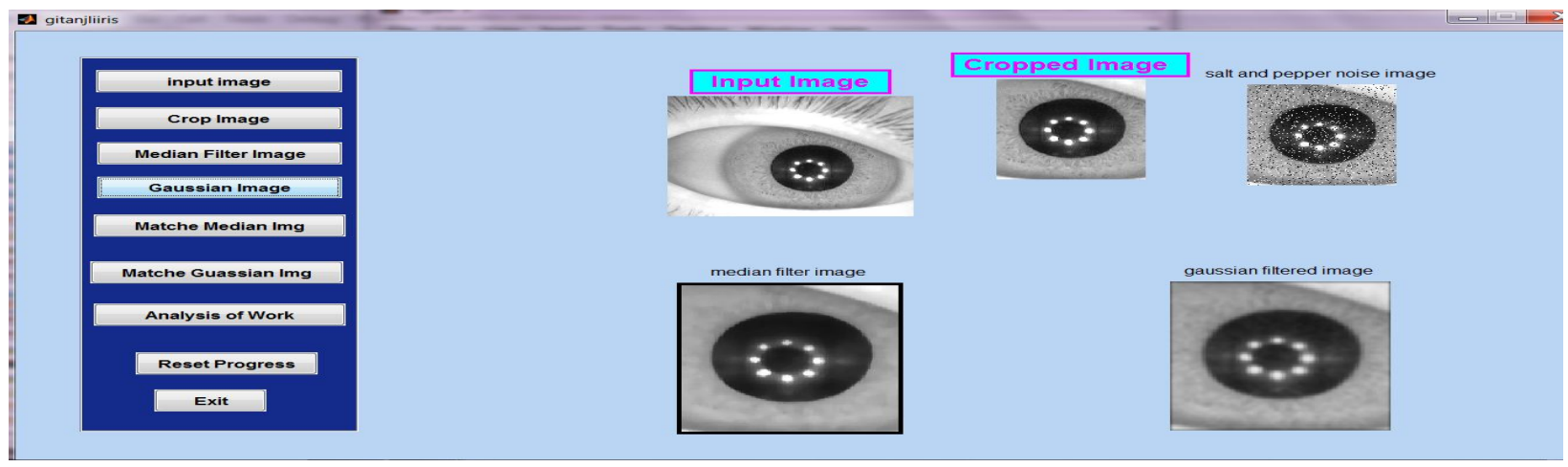

Figure 2: GUI layout of proposed work. 


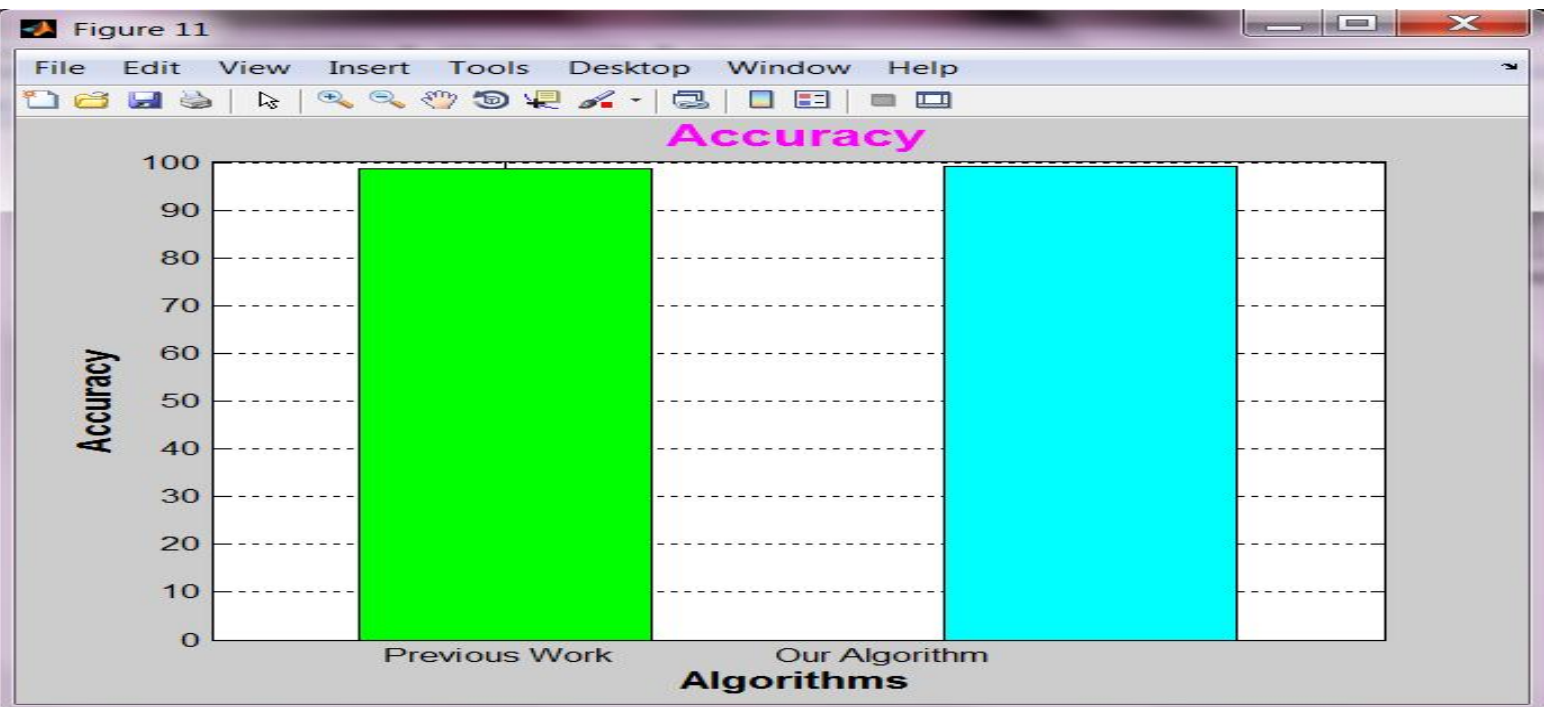

Figure 3. Comparison of accuracy of previous system and proposed system

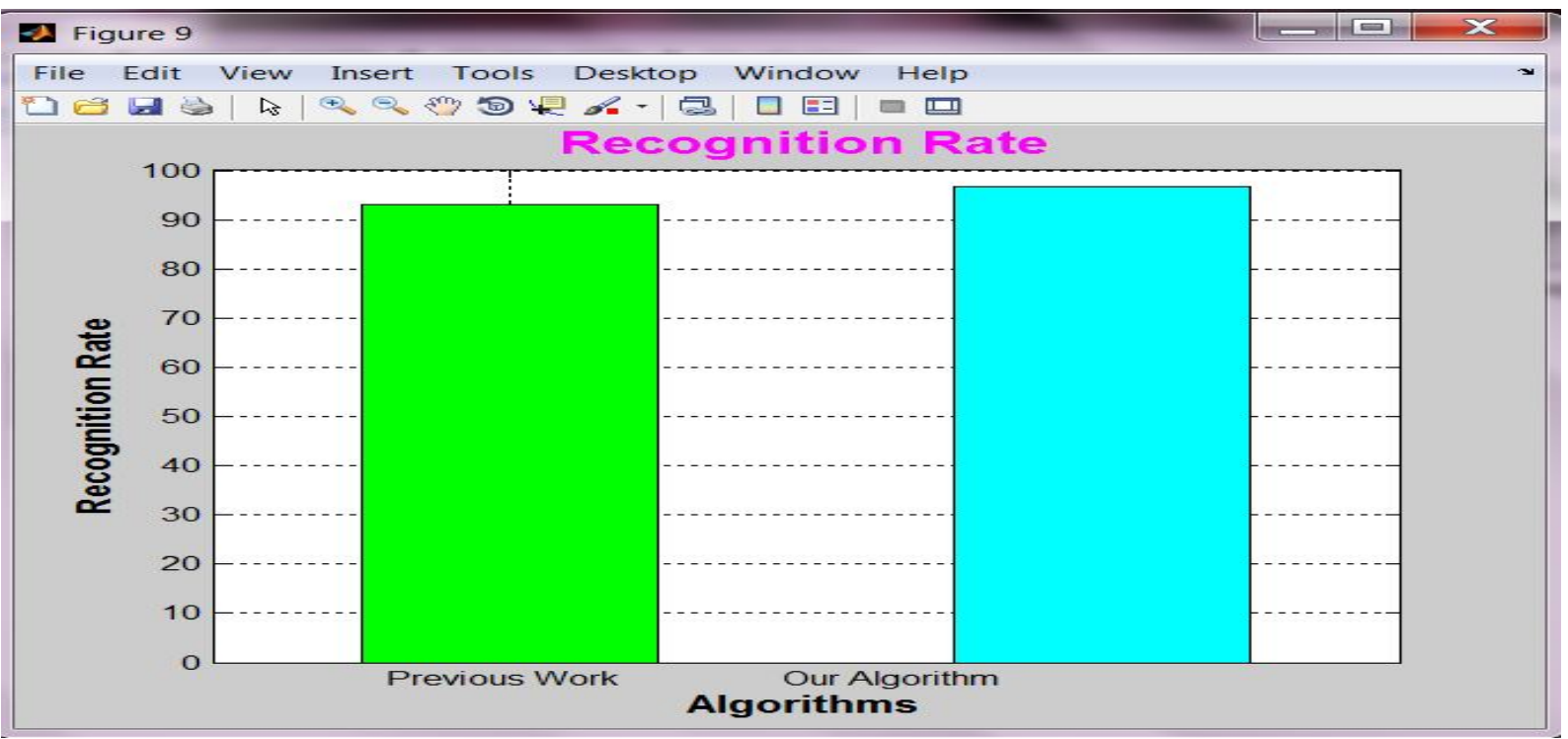

Figure 4. Comparison of recognition rate of proposed work and previous work

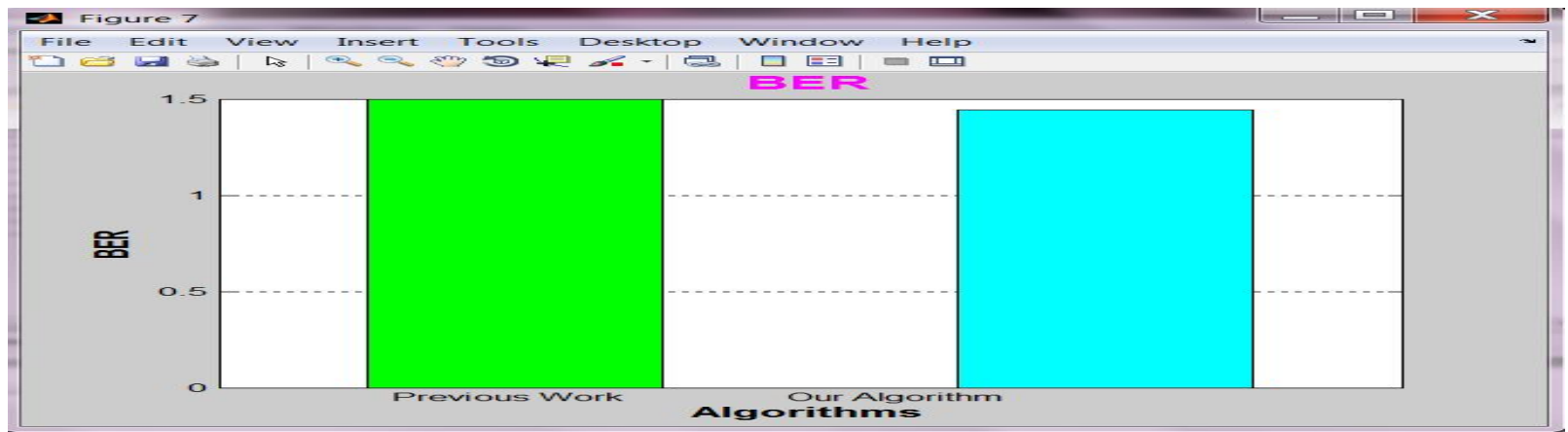

Figure 5. Comparison of bit error rate of previous work and proposed work 


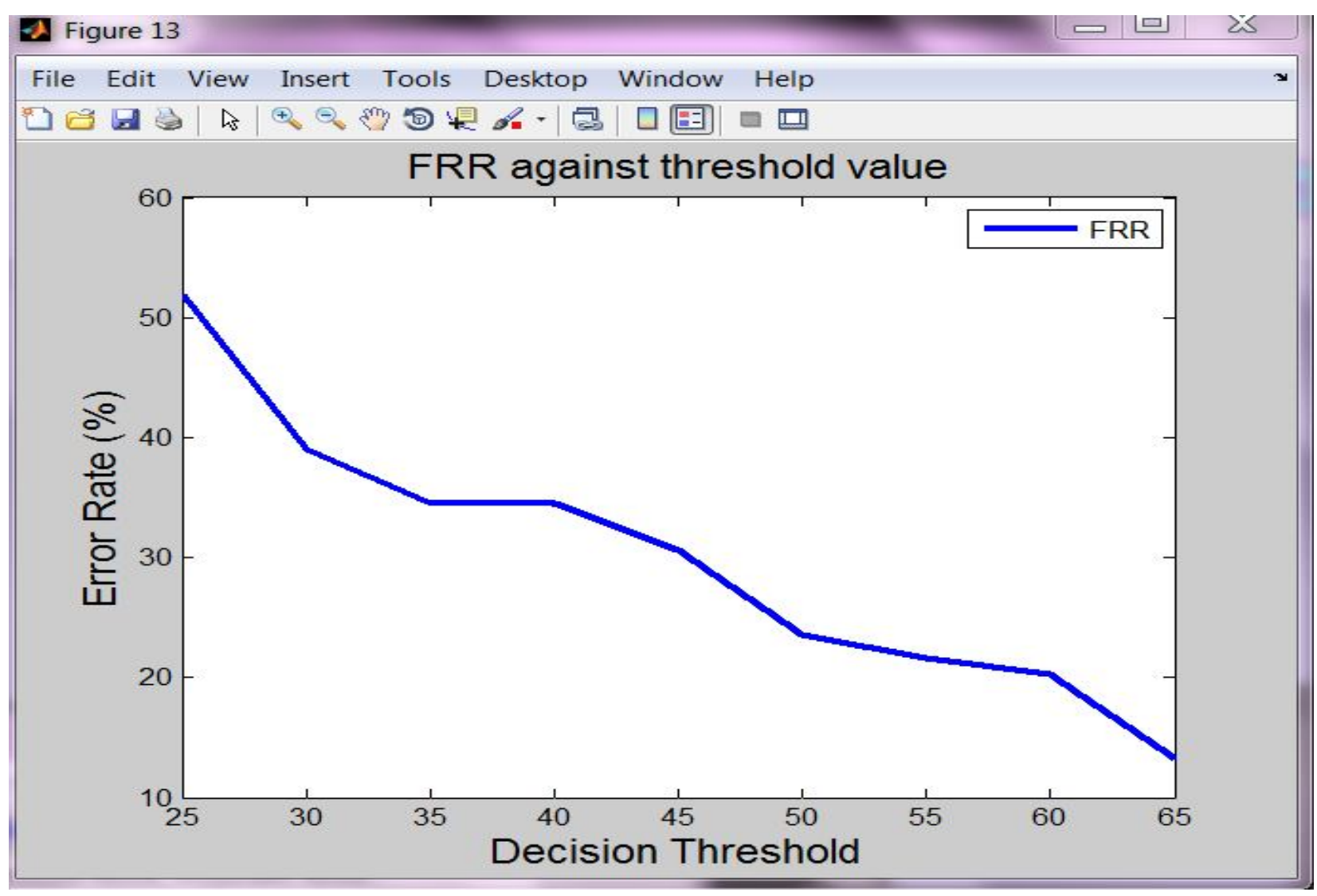

Figure 6. FRR curve against threshold value.

It can be illustrated from the results that if median filter is used in iris based recognition system rather than the guassian filter, the results are much better. Both recognition rate and accuracy of the proposed system based on median filter is better than the previous system based on guassian filter. Whereas, the bit error rate of the proposed system is lesser than the previous system. So, it can be said that iris based recognition using median filter is a promising solution to the identification problem.

\section{References:}

[1] Gerald Williams http://www.argus-solutions.com/pdfs/irisrecogwilliams.pdf , Iris recognition technology, iridian technologies, inc. 2001.

[2] Chedekel, M.R. "Photophysics and photochemistry of melanin. In Melanin: Its Role inHuman Photoprotection", Valdenmar, Overland Park, 11-23,1995.

[3] Vanaja Roselin.E.Chirchi, Dr.L.M.Waghmare, E.R.Chirchi, "Iris Biometric Recognition for Person Identification in Security Systems", International Journal of Computer Applications (0975 - 8887), Volume 24-No.9, June 2011

[4] L.M. Waghmare, S. P. Narote, A.S. Narote, "Biometric Personal Identification Using IRIS", Proceedings of International Conference on Systemic, Cybernetics and Informatics, Pentagram Research Centre - Hyderabad, pp. 679-682, Jan 2006 
[5]E. Wolff, “ Anatomy of the eye and orbit”, H. K. Lewis, London, UK, 7th edition, 1976.

[6] Yngzi Du, Robert Ives, Delores Etter, Thad Welch, Chein Chang, "A new approach to iris pattern recognition” In SPIE European Symposium on Optics/Photonics in Defence and Security, 2004.

[7] J. Mira, J. Mayer, “ Image feature extraction for application of biometric identification of iris - a morphological approach", In IEEE Proceedings of the XVI Brazilian Symposium on Computer Graphics and Image Processing (SIBGRAPIO3), 2003.

[8] Jaemin Kim, Seongwon Cho, Jinsu Choi, “ Iris recognition using wavelet features” In Kluwer Academic Publishers, Journal of VLSI Signal Processing, no. 38, pages 147-156, 2004.

[9] http://homepages.inf.ed.ac.uk/rbf/HIPR2/median.htm 